\title{
Los camisas doradas
}

\author{
Ricardo Pérez Montfort ${ }^{1}$
}

\begin{abstract}
$E_{1}$ 21 de noviembre de 1935 los encabezados de los principales diarios de la ciudad de México se ocuparon de "... los sangrientos choques entre radicales y 'Camisas Doradas'..." que habían sucedido el día anterior. El Universal afirmaba que los resultados del zafarrancho habian sido de tres muertos y 50 heridos. Dos días antes, el 19 de noviembre, la organización llamada Acción Revolucionaria Mexicanista mejor conocida como los Camisas Doradas habia anunciado que participaría en los desfiles del 25 aniversario del inicio de la Revolución mexicana. En la nota periodística de ese mismo día se decía que "... Los miembros de la ARM pedirán al general Cárdenas que baje al centro del Estadio (donde se desarrollarían los festejos) con el objeto de hacer en su presencia la jura de la bandera..."2 Esta invitación y la noticia de que participarian en los festejos fue suficiente para que protestara el Comité de Defensa Proletaria compuesto principalmente por organizaciones obreras, entre las que se contaban la Confederación General de Obreros y Campesinos Mexicanos, la Alianza de Uniones y Sindicatos de Artes Gráficas, el Sindicato Mexicano de Electricistas, la Alianza de Tranviarios, la Federación de Sindicatos Obreros del D. F., el Frente Unico del Volante, la Confederación Sindical Unitaria, el Sindicato Nacional de Ferrocarriles y los Obreros Metalúrgicos. En El Nacional se.comentaba que "... el solo anuncio propalado por la organización llamada 'Camisas Doradas' de que participará en el desfile en honor a la Revolución, causó en el seno de sindicatos y de centrales confederadas intenso disgusto, iniciándose desde luego gestiones de todo orden ante las autoridades federales y locales, a efecto de que dicho desfile no pueda llevarse a cabo". ${ }^{3}$ La razón de esta objeción consistía en que para el Comité de Defensa Proletaria los Camisas Doradas eran unos "provocadores de masas laborantes y del pueblo, rompehuelgas y terroristas". ${ }^{4}$ En un desplegado del mismo Comité dirigido a todos los trabajadores del D. F. se decía que les urgía que se concentraran en sus respectivos locales ya que las intenciones de los Camisas Doradas ese 20 de noviembre eran, según el Comité, " .... atacar los locales sindicales, romper las huelgas existentes y ejecutar otros actos de agresión en contra de las organizaciones de trabajadores..."5 En esos momentos las huelgas existentes eran las del Sindicato de Molineros y Empleados de Molinos
\end{abstract}

\footnotetext{
1 Este artículo es parte de una investigación mayor titulada "Los movimientos de extrema derecha durante el sexenio cardenista" que se lleva a cabo en el CIESAS (Centro de Investigaciones y Estudios Superiores de Antropología Social) bajo la responsabilidad del que esto escribe.

2 El Universal, 19 de noviembre de 1935, p. 5.

3 El Nacional, 20 de noviembre de 1935, p. 1.

4 Ibid, p. 1.

5 Ibid., p. 7.
} 
de Nixtamal, la de la Unión de Cargadores, la del Sindicato Unico de Trabajadores de la Industria Papelera y, en Chihuahua, una huelga minera cuyos representantes se encontraban en México en negociaciones. ${ }^{6}$

En efecto, tal como lo pronosticaron los planteamientos del Comité Nacional de Defensa Proletaria, tuvo lugar un enfrentamiento entre Camisas Doradas y grupos obreros. A eso de las dos de la tarde, cuando el zócalo se encontraba bastante lleno de manifestantes que festejaban el aniversario del inicio de la Revolución mexicana, se aproximaron a la explanada unos 75 jinetes y aproximadamente cien hombres a pie, vestidos con sus camisas amarillas o doradas, con sombreros que recordaban a las huestes de Pancho Villa, con banderas y pancartas que anunciaban la presencia de la Acción Revolucionaria Mexicanista. El secretario particular del presidente Cárdenas, Luis I. Rodríguez, se encontraba en el balcón central de Palacio Nacional, pasando revista a los contingentes, en representación del propio presidente. Bajo el balcón central “... se habían establecido como 30 coches todos manejados por choferes del Frente Unico de Trabajadores del Volante." Las carrocerías de los automóviles servían de tribunas improvisadas a los elementos radicales entre los que destacaba David Alfaro Siqueiros, quien en su discurso pidió a gritos al licenciado Rodríguez que se prohibiera el paso a los camisas doradas. En el tumulto se escucharon los gritos de "¡Ya vienen los dorados! ¡Mueran los fascistas vendidos a la burguesia!"7 El licenciado Rodríguez da entonces instrucciones para que el contingente de la ARM no pase frente a Palacio pero “... por el desacato a esta disposición el choque se produjo con los ánimos caldeados en ambos sectores manifestantes..."8 Los autos estacionados frente a Palacio Nacional encendieron sus motores y se lanzaron contra los jinetes que azuzaban a la gente con sus lazos. Al toque de un clarín los camisas doradas embistieron con palos arrancados de las gradas a los miembros de las organizaciones obreras y no tardaron en sonar unos balazos, que por cierto estuvieron a punto de herir al licenciado Rodríguez. Durante el zafarrancho, Nicolás Rodríguez, jefe supremo de los Camisas Doradas resultó herido "por instrumento punzocortante en el abdomen"9 y la Cruz Roja alcanzó a recoger a 34 heridos. La policía intervino y la contienda terminó con un saldo, según El Universal, de tres muertos y 50 heridos y según El Nacional de dos muertos y 47 heridos. De acuerdo con el primer periódico, "a los dorados los levantaron sus propias ambulancias, pues hay que advertir que llevaban en la columna un cuerpo completo de enfermeras, camillas, etcétera"'. ${ }^{10}$

En los tres días siguientes, la policía informó que "había detenido a diez Camisas Doradas que venían armados a la manifestación y que habían carecido de permiso para participar en el desfile, y que no obstante saber que podria haber fricciones, lo llevaron a cabo". ${ }^{11}$ El Comité de Defensa Proletaria protestó enérgicamente por los hechos ocurridos y realizó una manifestación luctuosa por dos de sus muertos, el día 24 de noviembre. Pidió además castigo para los responsables y la disolución de los "dorados". La cuestión llegó hasta el senado y el 22 de noviembre, Ernesto Soto Reyes, líder del bloque de senadores revolucionarios

6 El Universal, 20 de noviembre de 1935, p. 1.

$\rightarrow$ lbid., p. 1.

${ }^{8}$ El Nacional, 20 de noviembre de 1935, p. 1.

9 Ibid., p. 1.

${ }^{30}$ El Universal, 21 de noviembre de 1935, p. 1.

11 Ibid, p. 1. 
y representantes del estado de Michoacán, junto con Guillermo Flores Muñoz, también senador, condenaron a los Camisas Doradas y pidieron que se formara una comisión que hablara con el presidente Cárdenas para disolver a la organización ARM. En su discurso, Soto Reyes decía que "Los Camisas Doradas - hombres de paja a quienes yo considero irresponsables - fueron creados por elementos políticos de administraciones pasadas, con la intención de que la Revolución en México diera una vuelta en retroceso y se inclinara hacia la derecha." 12 Además presentaba la solicitud de disolución de esta organización afirmando que no representaba ningún interés sindical o de trabajadores, por lo tanto no se contravenía ningún estatuto jurídico al pedir su prohibición. A principios de 1936 la comisión emanada del Senado consiguió que se disolviera la organización de los Camisas Doradas por decreto del mismo presidente Cárdenas. Así, los sucesos del 20 de noviembre de 1935 determinaron el fin de esta Asociación, por lo menos en sus actos públicos, ya que a partir de 1936 su labor fue eminentemente clandestina.

Pero ¿quiénes eran los Camisas Doradas?, ¿qué tipo de intereses perseguían? y ¿cuál es la historia detrás de este grupo? es algo que pretendemos aclarar aquí. Pocas son las referencias más o menos autorizadas que se ocupan de dicha organización y todas la definen en términos muy vagos. Se refieren a la Acción Revolucionaria Mexicanista casi en los mismos términos en que lo hace la prensa mexicana y estadunidense del momento. Hugh G. Campbell en su libro La derecha radical en México 1929-1949 nos da un resumen de lo que hasta 1976 (fecha de su publicación) se sabía de esta organización. Basándose en artículos del New York Times, el Universal, el Excélsior y La Prensa en materia hemerográfica, y en las obras de Carleton Millan, Fernández Boyoli, Dulles y Mario Gill, ${ }^{13}$ nos dice que "la ARM (fue) la primera organización de los derechistas seculares que apareció en los años treinta y que jamás fue excedida ni en tamaño ni en renombre por ningún otro grupo posterior". ${ }^{14}$ Indica el autor que se formó tomando como modelo a los Camisas Pardas y los Camisas Negras de Alemania e Italia y que, además de caracterizarse por un ferviente antisemitismo y un acendrado anticomunismo, "existen evidencias para suponer que la organización se formó a iniciativa de Calles con el objeto de mantener controlados a los trabajadores". "is Esta suposición se maneja asimismo en el discurso de Soto Reyes como ya se ha visto. Campbell también afirma que los "dorados" recibieron subvenciones de la legación alemana y de ciertos agentes japoneses. Además menciona que la Confederación Patronal de la República Mexicana "dio apoyo a la ARM para ayudar a una organización previamente establecida" en su afán de fomentar la opo'sición de derecha radical secular en los años treinta. ${ }^{16}$

En un trabajo anterior a la elaboración de este artículo hicimos una

12 El Nacional. 22 de noviembre de 1935, p. 1.

13 La mayor parte de los libros mencionados aluden a los "dorados" de manera muy tangencial y los mencionan como un grupo "fascista" a las órdenes de los políticos más reaccionarios del momento. Es claro que la finalidad de estos trabajos no es un conocimiento cientifico de la realidad mexicana, sino que en términos generales también buscan algún fín politico; los puros títulos de Fernández Boyoli y de Gill son bastante explicitos (véase la bibliogralia).

it Campbell, p. 50.

is Ibid., p. 51 .

16 lbid., p. 58. 
breve caracterización de la ARM en relación con sus vínculos con la legación alemana y en general como parte de unos movimientos de la clase media de los años treinta. A hí especificamos que se trataba de una organización semimilitarizada, dentro del clásico estilo de los grupos de choque con consignas anticomunistas. Para marzo de 1935 ya tenia en su haber una serie de ataques a las oficinas del Partido Comunista al que veía como el agente "cuya meta es remplazar el presente régimen de México por un sistema soviético contrario a nuestra Constitución y a nuestras costumbres". También mencionábamos en dicho trabajo que la ARM se confunde frecuentemente con otra organización llamada la Unión de Veteranos de la Revolución, que lo mismo ataca "las tendencias comunistas y socialistas" del régimen de Cárdenas, que las declaraciones anticardenistas de Calles. El estudio de esta unión estä todavia por realizarse. ${ }^{17}$

Antes de entrar en los nuevos aspectos de la ARM que hemos investigado recientemente, valdría la pena aclarar que es muy poco probable que ésta recibiera ayuda de la legación alemana directamente. Como se ha podido comprobar, los grupos de extrema derecha secular tenian sin cuidado a los representantes del Tercer Reich en México, ya que éstos pretendian "asegurar la neutralidad" de nuestro país para permitir la continuidad del intercambio comercial entre ambos países. Una acción como la de dar dinero a los Camisas Doradas hubiera entorpecido dicha continuidad. Sin embargo no negamos la influencia ideológica del nazismo en la ARM.

En términos generales, así es la visión que se tiene de los Camisas Doradas. En ciertos aspectos esta visión parece mucho más simple de lo que hasta hora hemos podido investigar.

Recientemente fue abierto el archivo del general Francisco J. Múgica que hemos podido revisar. Además de otros documentos valiosísimos que no tardarán en arrojar luz a innumerables incógnitas que permean no únicamente el régimen de Lázaro Cárdenas, sino el del siglo $\mathrm{XX}$ mexicano en general, encontramos una gran cantidad de información sobre la ARM. ${ }^{18}$ Es probable que después de la disolución de este grupo, su archivo haya sido confiado al general Múgica, ya que quizá era el hombre de mayor confianza del general Cárdenas. Esto también es posible inferirlo según el tipo de documentos encontrados, no sólo referentes a los Camisas Doradas sino a otros muchos temas que hasta hoy no se han podido aclarar cabalmente por falta de documentación y. que no tienen por qué aparecer en el archivo de Múgica. Pero este es tema de otro ensayo.

La Acción Revolucionaria Mexicanista entra en escena en momentos en que el país se encuentra en una serie de reajustes políticos que valdría la pena mencionar antes de entrar en los detalles de la organización. El general Lázaro Cárdenas no llevaba ni un año de presidente de la República y ya había tenido que lidiar varios conflictos que exaltaban los ánimos de ciertos sectores del país. El 30 de diciembre de 1934 se había dado el enfrentamiento entre los Camisas Rojas de Garrido Canabal y los Feligreses de Coyoacán, con un saldo de cinco muertos.

17 El trąajo al que se alude es el de B. von Mentz, Verena Radkau, Daniella Grollova y R. Pérez Montfort, Los empresarios alemanes, el Tercer Reich y la oposición a Cárdenas, (en prensa).

is Esle archivo se encuentra en el Centro de Estudios sobre la Revolución Mexicana "Lálaro Cárdenas" en Jiquilpan, Michoacán. 
Los Camisas Rojas o el también llamado Bloque Juvenil Revolucionario era una organización creada en 1932 por jóvenes que asumian fervientemente los principales puntos del radicalismo ideológico de Garrido Canabal. Con un método muy ligado a la violencia, se mostraban en contra del fanatismo religioso, quemando santos y provocando a los enemigos del garridismo. Eran amantes pasionales de la educación nacionalista y del antialcoholismo, buenos marchistas, excelentes voceadores de consignas radicales y convencidos de los métodos agresivos para convencer a la población. Algunos Camisas Rojas portaban armas, desde los simples garrotes hasta las ametralladoras. ${ }^{19}$

Los sucesos de Coyoacán renovaron en buena medida los ataques de los grupos religiosos y de muchos otros grupos de civiles que se manifestaron abiertamente en contra del gobierno que permitía no sólo la existencia de grupos de choque, sino la clara preferencia que mostraba el secretario de agricultura, Tomás Garrido Canabal, por este tipo de acciones, las llamadas "reuniones dominicales antirreligiosas". ${ }^{20}$ En enero del año 1935 el arzobispo Pascual Díaz inició una serie de declaraciones que afirmaban que la persecución religiosa en México era peor que lo que había sido durante la rebelión cristera. Esto avivó los conflictos entre Iglesia y Estado, y durante la primera mitad de 1935 se llevaron a cabo los siguientes actos en contra de los grupos religiosos: en marzo fueron encarcelados el propio arzobispo Diaz y algunos de sus compañeros por oficiar sin autorización; la escuela socialista, en labios del secretario de educación, García Téllez, debía hacer todo lo posible por destruir el fanatismo implantado por la iglesia católica; Enrique Rodríguez, mejor conocido como "El Tallarín" encabezaba una "segunda cristiada" en el estado de Morelos que fue severamente reprimida.

Pero no solamente había conflictos en el terreno de la religión. La Universidad Nacional Autónoma de México también estaba inconforme con los postulados de la educación socialista y un conflicto en torno a los programas de estudio de la Escuela Nacional Preparatoria produjo en la Universidad gran animadversión contra el Estado.

En materia laboral, la agitación invadía los rincones más apartados de la nación. Para junio de 1935, alrededor de 1200 huelgas habían tenido lugar en toda la República. ${ }^{21}$ Desde Mérida hasta Tampico, de Veracruz a Uruapan y de San Luis Potosí hasta Chiapas, una gran actividad de las organizaciones obreras mantenia "en zozobra los círculos de inversionistas". ${ }^{22}$ La posición de Cárdenas al respecto era bastante clara. En sus apuntes decía que "Durante los movimientos de huelga últimamente registrados, los obreros no han estado inconsecuentes; se han colocado dentro de las posibilidades económicas de las propias empresas, que es la política del gobierno." 23

Esta actitud provocó para mediados del año de 35 las famosas declaraciones de don Plutarco Elías Calles que le valdrian su primer distanciamiento con el presidente Cárdenas y un año después la expulsión del país. Los conflictos entre legisladores callistas y cardenistas llegaron a sus puntos más álgidos en esta primera mitad del año. Además, el presidente también juzgó conveniente restructurar su gabinete. Eliminando

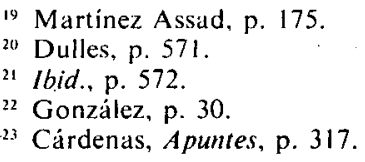


a los elementos callistas, Cárdenas nombró como sus colaboradores a hombres de su entera confianza. A partir de entonces el presidente concentró la responsabilidad de "la marcha politica y social de la nación" en su persona. ${ }^{24}$ Sin embargo, los conflictos estaban lejos de aminorarse. La Confederación Regional Obrera Mexicàna inició una gran campaña anticomunista en Tabasco, donde un grupo de garridistas arremetió contra un contingente de jóvenes que pretendían participar en la contienda electoral local, lo que provocó el inicio del desmonte del poder de Garrido Canabal; se suspendió la ayuda económica a la Universidad lo que también provocó gran incomodidad en ciertos sectores de la clase media y una balacera en la Cámara de Diputados dio como resultado la expulsión de 17 miembros de corte callista del Palacio Legislativo.

En las calles de la ciudad de México seguían deambulando las manifestaciones en apoyo a las huelgas existentes y las protestas por los acontecimientos internacionales que violaban la soberanía de los pueblos. El Frente Popular Antimperialista organizó un plantón frente a la legación italiana cuando se supo de la invasión a Etiopia y en varias ocasiones amanecieron las paredes de la legación alemana con pintas antinazis.

En cuanto a las organizaciones obreras, la fisura en la elite del poder habia fomentado la creación del Comité Nacional de Defensa Proletaria en donde participaban muchas confederaciones y sindicatos que pretendian defender sus derechos laborales e iniciar los trabajos para crear la Central Unica de Trabajadores. Este Comité apoyaba la política cardenista y criticaba los comentarios y tendencias desestabilizantes de los callistas. A la cabeza del comité se encontraban Fernando Amilpa y Vicente Lombardo Toledano, quienes como representantes de la Confederación General de Obreros y Campesinos Mexicanos se encontraban en pugna con la misma CROM y su secretario Luis Napoléón Morones, renombrado callista, que no tardaría en salir expatriado.

Así las cosas, la Acción Revolucionaria Mexicanista se aboca a la tarea de agitar el ya de por sí meneado ambiente público nacional.

Esta organización, fundada en el año de 1934, tenía como "Jefe Supremo" a Nicolás Rodriguez, que se decía antiguo villista y que había participado en varias rebeliones en el periodo posrevolucionario mexicano, destacándose en la del general Gonzalo Escobar en 1927. También había participado en la campaña vasconcelista de los años 28 y 29 , y durante el maximato organizó un primer ensayo de lo que serian los Camisas Doradas. Bajo la protección de algún funcionario callista, si no es que del propio Calles, Rodríguez creó los Camisas Verdes que se disolvieron al asumir el poder el general Abelardo L. Rodriguez. Con estos "verdes" se inició la campaña de "México para los mexicanos" que continuaría con los "dorados". ${ }^{25}$ La campaña consistió, fundamentalmente, en apalear a comunistas y judios. A los primeros los atacaban incluso en sus oficinas y en varias ocasiones fueron golpeados por dirigir o participar en huelgas o movimientos laborables; a los segundos los atacaban desde la prensa y publicando pequeños folletos que mucho recordaban los mecanismos de propaganda nazi. ${ }^{26}$

En los documentos relativos a la ARM encontrados en el Archivo

24 González, p. 44.

25. B. von Mentz, et al., op. cit., p. 14.

${ }^{26}$ lbid. 
Múgica, también se pueden leer algunas cartas que esta organización enviaba a ciertos judíos de la ciudad de México intimidándolos y presionándolos para que dejasen que "México sea para los mexicanos". Además una lista bastante larga de direcciones y nombres de judios fue enviada a todos los jefes de zona para que procediesen según los planes de la asociación.

La conformación de los Camisas Doradas se llevó a cabo, como ya deciamos, a principios de 1934, fundamentalmente entre miembros poco destacados del ejército nacional. En un principio la tarea de organizar, dirigir mensajes y nombramientos y en general las tareas básicas de la organización fueron llevadas a cabo por el propio Rodríguez. Para mayo de 1934, la ARM estaba dividida en 15 zonas repartidas por toda la República, pero principalmente concentradas en la ciudad de México, donde estaba su casa matriz. Estas zonas estaban formadas por varios grupos de diez a quince individuos. Cada grupo tenía su jefe que únicamente rendia informes al jefe de zona que a su.vez sólo rendía informes al "jefe supremo". Según datos de 1935, la ARM contaba con 350 jefes de grupo en las 15 zonas que habia establecido para su organización. Esto nos da un número probable de 3500 a 4000 miembros que se identificaban con los principios de los "dorados". ${ }^{27}$ Afirmaban que para ese mismo año ya podían formar un contingente de 5000 individuos. ${ }^{28}$ Estos, como decíamos, eran principalmente militares que en muy pocos casos ascendian a los grados de general o coronel; la mayoria eran capitanes, tenientes o sargentos, y no todos estaban en funciones dentro del ejército. Muchos de ellos ingresaban a la Acción Revolucionaria Mexicanista porque creian que Rodríguez podria interceder a su favor para reingresar al ejército.

Las distintas restructuraciones del ejército que se habian llevado a cabo desde el régimen de Obregón hasta el "maximato" habian dejado un gran saldo de militares sin ocupación y sin reconocimiento oficial. En el trabajo anteriormente citado nos referimos a este grupo que si bien no era de militares de baja graduación como infantería, "juanes" o soldados rasos, tampoco pertenecía a la elite militar emanada de la Revolución. Eran una especie de "clase media" militar que se había quedado bailando entre los beneficios que la Revolución había otorgado a ciertos militares encumbrados y el favorecimiento al ejército llano que Cárdenas llevaba a cabo como parte de sus reformas. Un amplio sector de estos militares clasemedieros parecían formar parte de asociaciones que pretendieron conseguir que se les reconociera como parte del ejército y así entrar al presupuesto. Algunas asociaciones se formaron con un claro tinte político, como la Unión Nacional de Veteranos de la Revolución o la misma ARM, y otras sólo se organizaron para lograr su ingreso al ejército, como la Asociación Nacionalista Mexicana o la Unión de Revolucionarios Agraristas del Sur. ${ }^{29}$

Otro fuerte contingente de esta organización pertenecía o perteneció a la política, tanto capitalina como veracruzana o de Guadalajara y Monterrey. Los mismos Camisas Doradas se jactaban de estar muy bien parados con las "fuerzas del orden público" en los siguientes estados: Chihuahua, Tamaulipas, Nuevo León, Coahuila, Aguascalientes,

${ }^{27}$ Archivo F. J. Múgica, vol. 107, p. 33.

${ }_{28}$ Archivo F. J. Múgica, vol. 107, p. 35, cfr. Rodríguez/Cedillo sept. 1935.

29 Pérez Montfort. Guía del archivo de Jenaro Amezcua, CEHM-Condumex, 1980, p. 17. 
Sinaloa, Durango, Zacatecas, Puebla, Jalisco, Guerrero, Michoacán, San Luis Potosi, Veracruz y Guanajuato. ${ }^{30}$ Además el general Vicente González, jefe de la policía capitalina, parecía estar en estrecha relación con el propio Rodríguez. Sin embargo quien los ve con buenos ojos y pretende protegerlos desde su nueva posición de secretario de agricultura a partir de junio de 1935 es el general Saturnino Cedillo.

Este hombre fuerte de San Luis Potosí, a pesar de haber apoyado a Cárdenas tanto en su candidatura como en sus primeros distanciamientos con el general Calles, se mantenía en constante reticencia frente al gobierno de don Lázaro. Parecía que el no haberlo incluido en el primer gabinete lo había dejado muy molesto. En enero de 1935, Cárdenas envió al general Múgica, entonces secretario de economía, a entrevistarse con Cedillo "... a comunicarle que su actitud de descontento está sirviendo de apoyo al clero y demás elementos reaccionarios en la labor sediciosa que éstos vienen desarrollando dentro y fuera del país..."31 Sin embargo, los acontecimientos en torno a las declaraciones de don Plutarco que significaron el reordenamiento del gabinete, dieron a Cárdenas la oportunidad de controlar con mayor eficiencia las actividades sediciosas de Cedillo. Al nombrarlo secretario de agricultura no sólo lo sacaba de su territorio, disminuyendo su posible influencia local, sino que le daba por su lado, satisfaciendo su ambición con un puesto de poca relevancia, ya que los conflictos en el agro, la distribución de la tierra y la reforma agraria principalmente, eran responsabilidad del Departamento Agrario y no de la Secretaria de Agricultura.

Desde abril de ese año, Rodríguez, nombrándose presidente del Consejo Patrio de la ARM se cartea con Cedillo no sólo para invitarlo a sus actos para "enardecer el fervor que tenemos [la ARM] en favor de nuestra enseña patria" sino para que forme parte de la misma organización. Rodriguez le escribe a Cedillo: “... lo invitamos porque deseamos vivamente contarle entre los más destacados miembros de esta organización ya que ideológicamente nos liga una idéntica afinidad..." 32

Los Camisas Doradas hacen una guardia de honor al general Cedillo cuando llega a la capital a ocupar su nuevo puesto en el gabinete; y a las dos semanas de que el potosino fuera nombrado secretario de agricultura del recién formado gabinete cardenista, Rodríguez le insiste "...y anhelando cristalizar mis promesas en realidad, he trabajado sin descanso en la organización de los 'dorados', para ofrecerle a usted en el plano ideológico y en todos los terrenos un contingente digno de tomarse en cuenta..." 33 Desde luego, Rodríguez aplaude su ingreso al nuevo gabinete y le recuerda la amistad que los liga, reconociéndolo como su jefe y amigo. También le dice: "Existe un enemigo poderoso que no pierde la ocasión de herirnos: el comunismo judaizante que está en el comercio, en la industria, en las instituciones bancarias y hasta en las esferas oficiales..." 34

Cedillo corresponde discretamente a las ofertas de Rodriguez. No lo mantiene demasiado cerca, pero tampoco permite que se aleje. Los meses de julio y agosto se pasan sin actividades relevantes. Sin embargo, en septiembre (mes de la patria) los "dorados" tienen intenciones de

${ }^{30}$ Archivo F. J. Múgica, vol. 106, p. 192.

31 Cárdenas, Apuntes, p. 312.

32 Archivo F. J. Múgica, vol. 107, cfr. 2672 c.p.

33 Ibid., cfr. 29 de junio de 1935.

34 Ibid. 
festejar un aniversario más de uno de sus máximos valores: la patria. Rodriguez escribe entonces a Cedillo una carta por demás reveladora: además de afirmarle que tiene interés en formar parte de las próximas solemnidades patrias, le ofrece nuevamente 5000 "dorados" para que disponga de ellos en los desfiles. Pero también le plantea que en un futuro próximo la Acción Revolucionaria Mexicanista debe ser “...el verdadero frente único en defensa de la Patria, a pesar de las intrigas de nuestros enemigos soliviantados por el pseudocomunismo internacional y pagados por el oro judío..." ${ }^{35}$ y le pide cinco mil pesos para comprar tres mil camisas y tres mil sombreros para uniformar a su gente. Le dice: "este esfuerzo de su parte será otra prueba de sus anhelos por cuanto tienda al bienestar nacional y un singular motivo de gratitud para los Dorados, que en todas circunstancias sabrán demostrarla" 36

Para entonces las relaciones entre Cedillo y los "dorados" habian despertado sospechas de otros miembros del gabinete de Cárdenas, principalmente de Francisco J. Múgica quien se encargó de mandar vigilar tanto al potosino como a los "dorados". Varios memoranda confidenciales informan de entrevistas entre Cedillo y Rodríguez. Inciuso algunos mencionan que Cedillo está dándoles dinero a los "dorados" para comprar armamento. ${ }^{37}$ Sin embargo, todo parece indicar que las sospechas de Múgica no son tomadas demasiado en serio por los órganos de seguridad del gobierno cardenista, hasta que suceden los hechos del 20 de noviembre. A partir de entonces no solamente se actúa con energía en contra de la ARM sino que el mismo Cedillo empieza a enfrentarse constantemente a los demás miembros del gabinete, en especial al general Múgica, que no sólo lo tiene muy bien vigilado, sino que pretende no tolerar más actividades sediciosas dentro de la cúpula estatal.

Como ya se dijo, el general Múgica era el hombre de confianza del presidente Cárdenas en materia de control, tanto dentro como fuera del gabinete. Es Múgica quien tiene que lidiar con los conflictos iniciales de inconformidad por parte de Cedillo, y será él también quien mantenga al presidente al tanto.

El año de 1936 significa para las relaciones Cedillo-Rodríguez cierto distanciamiento. El primero pretende conservar su puesto en el gabinete y el segundo tiene que salir de la ciudad de México, una vez que los Camisas Doradas son disueltos legalmente, y expulsados después del país. Si bien Cedillo empieza a cuidarse un poco más, haciendo menos alarde de sus diferencias con el gabinete, Rodríguez y lo que queda de sus "dorados" siguen agitando y tratando de mantener a flote su lucha en contra del "comunismo judaizante".

Debilitados por el golpe asestado por el gobierno cardenista, los "dorados" tratan de asociarse con otros grupos que puedan congeniar con sus intereses; a pesar de ello los intentos por encontrar afinidades se ven obstaculizados constantemente. Según informes presentados ante Múgica, durante el año de 36 "se llevan a cabo reuniones en las que participan 'dorados', antirreeleccionistas, vasconcelistas, cristeros $y$ otros desafectos al gobierno... ${ }^{38}$ pero el constante desacuerdo en cuanto a tácticas, fechas de acción y posibles liderazgos no permite que

3s Ibid., cfr. 2 de septiembre de 1935.

36 Ibid.

37 Ibid., vol. 106, p. 200.

38 Ibid. 
se lleven a cabo acciones conjuntas.

Bastante decepcionado por esta situación, Rodríguez empieza a cartearse con Henry Allen de los Estados Unidos. Este personaje es el jefe del Silver Battallion, grupo anticomunista y antijudio de California. Allen invita a Rodríguez a Los Angeles, ofreciéndole protección y afirmando que "su lucha es nuestra lucha." 39 La reunión parece preocupar tanto al gobierno estadunidense como al mexicano; la Secretaría de Guerra de nuestro país pide informes al cónsul mexicano sobre Allen, y a principios de 1937 se envía un mensaje en el que se dice que la reunión entre Allen y Rodríguez puede llevarse a cabo en Guaymas. La entrevista entre el "plateado" y el "dorado" no parece haberse concertado, fundamentalmente porque Allen no consiguió "forma alguna" de entrar al pais. Más tarde, Rodríguez intentará ponerse en contacto con él en los Estados Unidos. Pero la distancia entre San Antonio Texas, que es donde Rodriguez tendrá su centro de actividades a partir de la segunda mitad del año de 37, y Los Angeles, no permitirán ninguna reunión entre los dos anticomunistas.

Mientras tanto, el distanciamiento entre Saturnino Cedillo y el presidente Cárdenas se hará mucho más patente ese mismo año, hasta que en agosto un pequeño conflicto en la Escuela de Agricultura de Chapingo provocará la renuncia del potosino, quien ni tardo ni perezoso regresará a San Luis para encontrarse con la necesidad de reforzar sus instrumentos de poder local. La renuncia de Cedillo coincide con la publicación de un manifiesto firmado por Rodríguez, dirigido a los jefes de zona, a los jefes de grupo y a los "dorados" en general para que "templen sus espíritus de la fortaleza y del entusiasmo que la Patria les exige y que, cuando llegue el momento de decir 'a la lucha compañeros', todos como una falange de titanes respondamos con el grito de PRESENTE." 40

El manifiesto parecia indicar que todo estaba listo para apoyar una rebelión que debería acaudillar el propio Cedillo. Sin embargo, éste tardó mucho en organizarse y cometió un gran número de torpezas. ${ }^{41} \mathrm{La}$ mayor parte de sus movimientos eran conocidos por el general Múgica, quien había logrado intervenir las comunicaciones en el estado de San Luis Potosí e informaba constantemente tanto al presidente como al secretario de guerra de lo que acontecía.

Los "dorados", principalmente el propio Rodríguez, hacian todo lo posible por ponerse en contacto con Cedillo sin lograrlo cabalmente. Parecían, o más bien parecía Rodríguez, estar esperando la orden de Cedillo para lanzarse a la lucha. Aquella fuerza de cinco mil hombres se habia diezmado dramáticamente desde la desbandada de principios del año de 36 y es probable que el propio Cedillo hubiese dejado de considerar a los "dorados" como un grupo lo suficientemente sólido como para apoyarlo en su próxima aventura. Los últimos informes que se tienen de los "dorados" datan de febrero y marzo de 1938, tres meses antes de que Cedillo se levantara en armas contra el régimen de Cárdenas. Dichos informes parecen más bien patadas de ahogado, puesto que llaman a la unidad, atacan el régimen de Cárdenas y sus "tendencias comunistas y extranjerizantes", pero sólo advierten la posibilidad

${ }^{39}$ Ibid., p. 300 .

40 Ibid., vol. 107, p. 369.

41 Pérez Montfort, Notas sobre la rebelión cedillista, Anuario del Colegio de Historia, Facultad de Filosofia y Letras, UNAM, 1984. 
de llegar a ser una fuerza, sin ninguna afirmación de rebelión o confrontación armada contra el gobierno. Aquellos enfrentamientos callejeros de 1935, los desplantes de agresividad y acciones en conjunto daban paso a manifiestos un tanto cuanto incoherentes que anunciaban su falta de organización y fuerza.

Como es bien sabido, la rebelión cedillista se llevó a cabo en mayo de 1938 y en menos de dos meses quedó liquidada. Los "dorados" no volvieron a aparecer en la escena nacional y Rodríguez no regresó al país sino hasta la década siguiente.

Los dos últimos manifiestos son verdaderos portentos del discurso nacionalista de la extrema derecha. El llamado a la unión que hace Rodríguez en agosto del año de 37 está lleno de referencias a los valores clásicos de este discurso. Partiendo de un sistema de opuestos, lleno de verdades absolutas asumidas como un acto de fe que busca darles un sentimiento de identidad, el discurso se pasea a través del uso de adjetivos grandilocuentes. El trabajo, la familia, la patria, el honor, la propiedad, la moral y la bandera son piezas indiscutibles en el sistema de valores.

Pero vayamos por partes. Ya dijimos que un punto básico de la ideología de esta asociación es su anticomunismo. Ellos, los "dorados", van a representar todo "lo bueno" y el comunismo todo "lo malo". Los "dorados" son, según estos dos manifiestos: "un haz de corazones resueltos y de conciencias limpias... los hércules que salvamos el honor nacional... formamos una organización soberana y respetuosa de los derechos ajenos (sic) que por la firmeza de sus principios y de la honradez de sus procedimientos siembra el miedo entre las filas rojas y salvajes..." Y los comunistas son "los que congestionados de ambición y de maldad, se arrojan los unos a los otros, como hienas, para disputarse los bienes ajenos (sic), que son su único ideal..." son "la barbarie que intentó invadir no sólo las salas del presupuesto sino nuestros propios hogares..." son "las hordas rojas y salvajes..."

Los "dorados" representan la única salvación: "...no olvidemos que somos los abanderados de la justicia y de los derechos que nos quiere arrebatar un Estado irresponsable, como lo es el Estado comunista..." son "... los únicos que han mantenido en alto el pendón de la legalidad y no han dejado que estallen con los impulsos bestiales que acostumbran las oprobiosas vejaciones ni los abusos de la canalla roja ensordecida y respaldada por el actual gobierno o desgobierno que deshonra a nuestra Patria..."42

La razón por la que luchan los dorados es desde luego "la salvación de la Patria". "Su obligación es morirse antes que permitir se mancille el honor de las Instituciones y de la Patria". La defensa de su sistema de valores compuesto por la familia, la moral, la propiedad, etc., justifica toda acción en contra del enemigo: el comunismo.

Nuevamente la violencia se muestra como característica básica de este discurso, adosada con llamados a la virilidad y a la disciplina. El resultado es, pues, un discurso extraordinariamente agresivo que dentro de un nacionalismo concebido como "la expresión de una reacción frente a un desafío extranjero... que se considera una amenaza para la integridad o la identidad nativas" 43 se vuelve de ataque, más que de

42 Archivo F. J. Múgica, vol. 107, p. 369; t. 207 p. 277.

${ }^{43}$ David Brading, Los origenes del nacionalismo mexicano, México, Sep-setentas, 1973, p. 12 . 
consolidación, de apoyo o de defensa. Y es de ataque porque no parecen tener muy claro qué es lo que defienden; no tienen un programa de acción definido, solamente se saben anticomunistas, pero en términos irracionales; más por miedo que por convencimiento. Miedo a verse afectado por los cambios; en fin, miedo al cambio: reaccionario.

Los Camisas Doradas intentaron con este tipo de discurso llamar la atención de la clase media afectada por las reformas cardenistas, incluyendo aquellos miembros del ejército que en un momento dado habían apoyado e ingresado a las filas de estas organizaciones de extrema derecha. Una vez fracasado el golpe de Saturnino Cedillo y arreciado la reacción gubernamental en contra de los grupos sediciosos - a partir de 1938 se empiezan a desmoronar agrupaciones como la Confederación de la Clase Media, la Unión Pro-Raza, el Partido Antirreeleccionista y otros. Los miembros de estos sectores descontentos, solamente podrán satisfacer sus necesidades de oposición en la campaña almazanista. En ésta se asociará el Partido Revolucionario de Unificación Nacional. No se incorporarán a estos grupos: el Partido Acción Nacional y la Unión Nacional Sinarquista, ambos fundados en 1938. Este hecho todavía requiere mayor profundización en su estudio para encontrar una explicación satisfactoria. Sin embargo, es necesario mencionar que a pesar de encontrarse en una situación que más o menos favorecía la unión de los grupos de extrema derecha -desde los movimientos clasemedieros hasta los fanáticos religiosos - esta posible unión abortó claramente en 1938 con el fracaso de la rebelión cedillista.

\section{Bibliografia}

Campbell, Hugh, G., La derecha radical en México, 1929-1949, Sep-setentas, México 1976.

Cárdenas, Lázaro, Obras: Apuntes 1913/1956, UNAM, México 1972.

Dulles, John F., Ayer en México, FCE, México, 1977.

Fernández Boỷoli y Eustaquio Marrón de Angelis, Lo que no se sabe de la rebelión cedillista, Grafi $=$ art, México, 1938.

Gill, Mario, El sinarquismo, su origen, su esencia, su misión, Editorial Ollin, México, 1962

González, Luis, Los dias del presidente Cárdenas, COLMEX, México, 1981. 